\title{
A Self-powered Module with Localization and Tracking System for Paintball
}

\author{
Andrey Somov ${ }^{1}$, Vinay Sachidananda ${ }^{2}$, and Roberto Passerone ${ }^{1}$ \\ ${ }^{1}$ University of Trento, Trento, Italy \\ \{somov, roby\} adisi.unitn.it \\ ${ }^{2}$ Darmstadt University of Technology, Darmstadt, Germany \\ vinay@cs . tu-darmstadt.de
}

\begin{abstract}
In spite of the popularity of wireless sensor networks (WSN), their application scenarios are still scanty. In this paper we apply the WSN paradigm to the entertainment area, and in particular to the domain of Paintball. This niche scenario poses challenges in terms of player localization and wireless sensor node lifetime. The main goal of localization in this context is to locate and track the player in order to facilitate his/her orientation, and to increase the level of safety. Long term operation could be achieved by adopting appropriate hardware components, such as storage elements, harvesting component, and a novel circuit solution. In this work we present a decentralized localization and tracking system for Paintball and describe the current status of the development of a self-powered module to be used between a wireless node and an energy harvesting component.
\end{abstract}

\section{Introduction}

A Wireless Sensor Network (WSN) is a distributed collection of nodes which are resource constrained and capable of operating with minimal user attendance. Wireless sensor nodes operate in a cooperative and distributed manner. However, there are application areas, such as the entertainment domain, where WSNs are still seldom applicable. In this paper we apply the concept of WSN in a Paintball application. Paintball is a sport in which players eliminate opponents from play by hitting them with paint. For a successful Paintball application we have to solve two important problems: player localization and long term operation of the wireless sensor node.

At present, players which are involved in Paintball use the Global Positioning System (GPS) [6] for orienting, walkie-talkie to communicate with each other or do not use these devices at all in case of short-term and bounded space scenario. However, providing each of e.g. hundred players with an individual GPS receiver is not always the preferred solution for cost reasons. Moreover, GPS itself is a power hungry component and needs at least four known satellites to be visible in order to find the coordinates of the receiver.

Over the years, many protocols [1] have been devised to enable the location discovery process in WSNs to be autonomous and able to function independently of GPS and other manual techniques. In all cases, the focal point of location discovery has 
been a set of special nodes known as beacons, which have been referred to by some researchers as anchor, locator, or seed nodes.

As for long term operation, it has always been the key issue in power supply design for wireless sensor nodes. Energy harvesting components, such as solar cells or piezoelectric converters, have to some extent solved the lifetime problem. However, the availability of heterogeneous WSN platforms has resulted in a problem of inflexibility: it is difficult to exploit a wireless sensor node and a harvesting component together. Basically, WSN platforms are being developed for particular cases $[3,5]$ or have already been integrated with a harvesting component [2].

In this paper we propose a localization and tracking system solution that is an extension of the work based on the existing systems RADAR [26] and MoteTrack [25]. The proposed system does not rely upon any back-end server or network infrastructure. The location of each mobile node is computed using a received radio signal strength signature from numerous beacon nodes to a database of signatures that is replicated across the beacon nodes themselves.

To provide "perpetual" support of the hardware, we designed a mediator board between a wireless sensor node and a harvesting component. This board has a two-level energy storage buffer including two supercapacitors wired in series as a primary buffer and a li-ion battery with high capacitance as a secondary buffer. A novel circuit solution coupled with advanced electronic components is used to charge the supercapacitors and the li-ion battery safely to prolong their lifetime. Moreover, the board supports AC or DC based ambient power source that will make the entire WSN more flexible.

The paper is organized as follows: Section 2 will introduce a review of (a) recent localization systems, and (b) existing wireless sensor platforms with energy scavenging technology; Section 3 will present the main goals of the paper; Section 4 will describe the architecture of the self-powered module and the implemented selfpowered board, and finally, Section 5 will present the designed localization and tracking system with the experimental results.

\section{Background}

In this section we briefly review some of the existing and widely used location tracking systems, as well as wireless platforms with energy scavenging technology.

\subsection{Localization Systems Overview}

In Global Positioning System (GPS) [6], [13], triangulation [26], [16] uses ranges to at least four known satellites to find the coordinates of the receiver, and the clock bias of the receiver. The triangulation procedure starts with an a priori estimated location that is later corrected towards the true location. Due to high power consumption, relative inaccuracy and indoor operation instability it is undesirable to use GPS in WSN.

RADAR [26] is the first indoor localization system. It is based on empirical Received Signal Strength Indication (RSSI) measurements as well as a simple yet effective signal propagation model. RADAR uses a standard 802.11 network adapter to measure signal strength values with respect to the base stations that are within range 
of the network adapter. A drawback of this location approach is that the signal pattern is recorded statically in the database and may greatly differ from the values measured in the dynamic environment.

MoteTrack [25] is a decentralized localization system which is more sucessful in indoor localization and it is largely based on the RADAR [26] approach. The location of each mobile node is computed using RSSI signature from numerous beacon nodes to a database of signatures that is replicated across the beacon nodes themselves. MoteTrack can tolerate the failure of up to $60 \%$ of the beacon nodes without severely degrading accuracy, making the system suitable for deployment in highly volatile conditions.

Cricket [14] was designed and implemented as an indoor mobile decentralized navigation system called CricketNav [14]. It is based on Ultrasound and RF, and uses independent, randomized transmission schedules for its beacons and a receiver decoding algorithm that uses the minimum of modes from different beacons to compute a maximum likelihood estimate of the location. However, the CricketNav system requires large amount of extra hardware to be installed.

A distributed and leaderless algorithm [28] performs the detection of the logical location in specks. This method employs binary connection information for range estimation, as opposed to RSSI method applied in our work.

There are plenty of RSSI based localization systems, e.g. RADAR [26], MoteTrack [25], and commercial applications, for example Ekahau [27]. However, we have focused our attention on developing more robust and efficient RSSI based approach.

\subsection{Wireless Sensor Platforms with Energy Scavenging Technology}

In this section we will review recent wireless sensor platforms with energy scavenging technology.

VIBES [2] and PMG Perpetuum [21] are microsystems powered from ambient vibrations. VIBES is an energy aware system and has a possibility to adjust the duty cycle according to the available energy. PMG platforms have a primary energy buffer for improved flexibility and do not require any maintenance.

The next three wireless sensor platforms with energy scavenging technology, namely Heliomote [3], Prometheus [4] and Trio [5], contain off-the-shelf modules Mica2 [9] for the first one, and Telos [7] for Prometheus and Trio. In contrast to VIBES, Heliomote has solely NiMH rechargeable batteries as energy buffer. Prometheus is a wireless sensor platform which includes the Prometheus power board [4] and Telos. The entire system is being powered by the Prometheus power board. This power board is implemented with a two-stage storage system containing two supercapacitors as primary buffer and a lithium rechargeable battery as secondary buffer. Prometheus, Telos and XSM [8], in turn, are combined into the Trio node. Telos is necessary for low power operation, Prometheus is responsible for power supply, and finally XSM is a set of indispensable sensors.

However, all the listed platforms support either DC (solar radiation) or AC (vibration) ambient power source that leads to inflexibility of the entire WSN. 


\section{Key Principles of the System}

The most well-known and popular Paintball types are speedball, woodsball and scenarioball. The scenarioball type appears to be most interesting for our research.

The playing field in scenario paintball (the storyline can be anything like civil war events, gangster wars of 1930s, the Oklahoma D-Day, storming a building or rescuing hostages) is normally large and in most cases unknown for the players. Moreover, the game may last several hours, therefore it would be reasonable to provide the players with a wireless sensor node to track their position by beacon nodes deployed throughout the playing field. It could be done for safety reasons in the interests of a paintball player, and with the aim of assisting him/her in orientation by showing his/her location on a Personal Digital Assistant (PDA).

However, we would like to pay your attention to some important requirements and constraints of the localization system. First of all, the beacon nodes should be distributed over the playing field in such a way that the players are within the radio range to be tracked. The player location computation should be performed quickly to guarantee his relevant position. Since the playing field is in most cases a natural setting, the energy proves to be a limiting factor. The system accuracy depends on the signal power and frequency band, which, again, leads to energy restrictions. Thus, the main goal is to design an accurate and failure-tolerant long-term localization system for Paintball players tracking which is applicable to the indoor and outdoor operation.

The system requirements and our contribution are described in more detail in Section 4.1 and Section 5.

\section{Self-powered Module Design}

In this section we present the general block diagram of the self-powered module (see Figure 1), describe the implemented self-powered board (see Figure 2), and finally discuss the hardware selection.

\subsection{Self-powered Module Architecture}

In our design we apply a modular uniform technology for WSN with a power management technique. The entire system is to contain three main parts: the ambient power source $\rightarrow$ the power management board $\rightarrow$ the wireless sensor node. The ambient power source consists of a harvesting component converting ambient energy into electric energy. In other words, the self-powered board designed is an enhancement for wireless sensor nodes which provides interoperability with different types of harvesting components and long term operation for localization system.

The general block diagram of the self-powered board is depicted in Figure 1. The parts shadowed in grey refer to the self-powered module.

There are three power supplies in the entire self-powered system: an Ambient Power Source, a Supercap (Primary Buffer), and a Rechargeable Battery (Secondary Buffer). Since the Rechargeable Battery has the lowest lifetime, which mostly depends on the quantity of charge-discharge cycles, its energy is the most valuable. The Ambient Power Source is inexhaustible, but unstable. Thus, it is more reasonable to 
exploit the Ambient Power Source energy by charging Supercap using Voltage Stabilization. This means that the entire system must operate at first from the Primary Buffer. When the Supercap is discharged, the system is to use the Rechargeable Battery as power supply. After being discharged, the Secondary Buffer must be replenished with Supercap energy. The wireless sensor node microcontroller must monitor the energy level of the power supplies via the Voltage Monitor of the Supercap and the Charge/monitor controller of the Li-ion battery and manage the Analog Switch in accordance with the energy availability of power supplies. This solution will increase the lifetime of the energy storage devices.

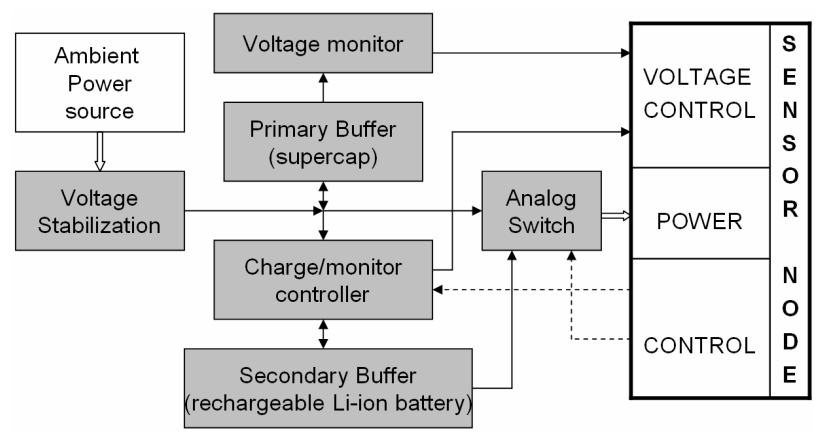

Fig. 1. The general block diagram of the self-powered module

The current flexible solution will allow one to connect a wireless sensor node with a harvesting component which operates using an environment energy source like solar rays, vibration or temperature difference. A user just needs to choose an appropriate wireless sensor node which suits according to cost and adequate ambient power source for the application. Moreover, the schematics proposed enables two storage buffers to support power hungry applications and to increase the lifetime of the sensor node. Voltage monitors allow the MCU to see how storage buffers are charged.

The major distinguishing feature of the self-powered board with respect to traditional energy scavenging technology is the possibility of charging its energy buffers either from DC or AC ambient power sources by switching a slide switch for the appropriate source. The self-powered platforms observed in Section 2 are able to operate with a preset energy scavenging component.

\subsection{Hardware Selection}

The self-powered board is shown in Figure 2. Its size $(40 \times 70 \mathrm{~mm})$ is similar to usual wireless sensor nodes. We have marked out at the top and bottom layers of the board the pads of the main parts according to the general schematics sketched out in Figure 1. Since our flexible design makes it possible to apply different kinds of ambient power sources, the first component is a slide switch which performs switching between AC or DC options. The self-powered module supports a $1.3 \mathrm{~W}$ ambient power source. AC voltage is converted into DC voltage by a diode rectifier DB102S and an 
electrolytic capacitor which are standard components for this case. Then the zener diode BZV85C4V7 stabilizes the DC voltage up to 4.7V.

We used two supercapacitors wired in series to reduce leakage current as a primary energy buffer. The supercapacitors by Nesscap EDLC [24] with $2.3 \mathrm{~V}$ maximum voltage rating are an excellent fit for our design. Besides, we applied a resistor in series with the $4.7 \mathrm{~V}$ stabilized DC voltage source to charge the primary buffer with an appropriate voltage. In line with [4], we have chosen $25 \mathrm{~F}$ capacitance for each of the supercapacitors.
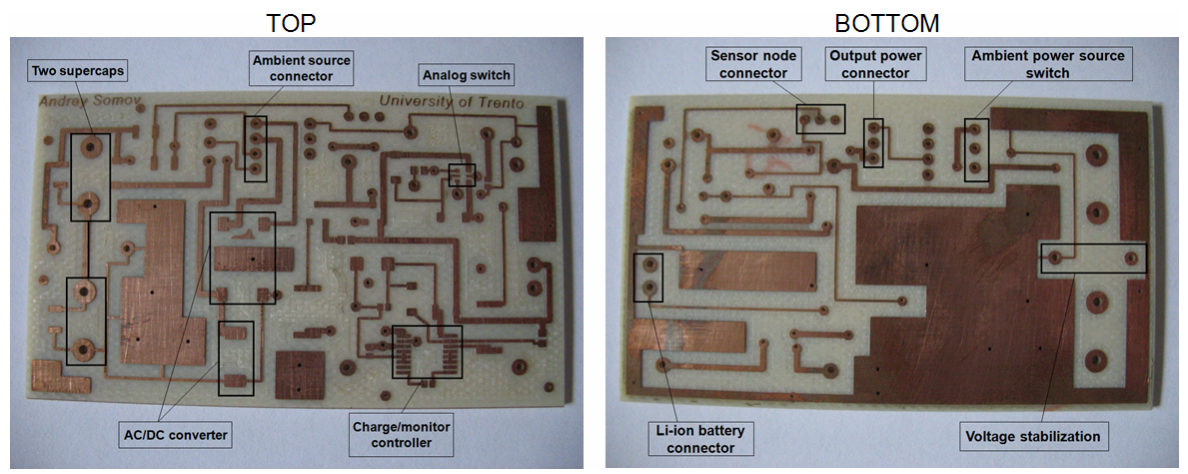

Fig. 2. The $40 \times 70 \mathrm{~mm}$ self-powered board

Panasonic [11] CGR18650E Li-ion rechargeable battery, with 3.7 nominal voltage and $2550 \mathrm{mAh}$ typical capacity, acts as a secondary energy buffer. Lithium rechargeable batteries have no memory effect while they provide high charge-discharge cycle, highest density and lowest leakage [4]. However, they require a more complex charging circuit like, for example, a special charge controller.

To charge the secondary energy buffer properly we applied a DS2770 battery monitor and a charge controller [29]. This integrated circuit performs several functions needed for thorough battery maintenance. The secondary buffer is charged by the primary buffer, but when the primary buffer is being charged and the ambient power source provides a stable voltage, then it is possible to charge the secondary buffer directly.

We used the Single Pole Double Throw (SPDT) switch ADG849 [18] to choose either the primary or secondary power buffer to supply the wireless sensor node. ADG849 has high current carrying capability and low power consumption.

The self-powering module enables $3.3 \mathrm{~V}(1 \mathrm{~W}, 276 \mathrm{~mA}$ maximum current) output voltage by applying the zener diode $1 \mathrm{~N} 4728 \mathrm{~A}$ which stabilizes the voltage up to this magnitude.

For interconnection between the self-powered module and the wireless sensor node we applied a 3-pin connector for charge/monitor battery control, supercapacitor voltage monitoring, and analog switch control. Another 3-pin connector carries the power supply for wireless sensor node. A 4-pin ambient source power connector makes it possible to attach an external energy harvesting component. 


\section{Decentralized Localization Algorithm}

This section describes a new accurate and robust decentralized localization algorithm which supports database distribution. To obtain these results we made the following contributions. At first, the computation is completely done in a decentralized fashion to get a better localization system which works on both CC1000 [19] and CC2420 radios [20]. Moreover, there is no back-end server, so all the computation is performed in the deployed sensor motes. Secondly, the location signature database is replicated across the beacon nodes, similarly to previous systems [25], [26], but here the replication is done while dividing the database with the basic stable marriage algorithm. This minimizes per-node storage overhead and provides high robustness to failure and ensures more accuracy. Thirdly, there is a new memory management technique and queuing technique which is needed for the distribution of data and the location estimation process. Finally, we employ a dynamic radio signature distance metric which is quite similar to RADAR [26] and an idea from MoteTrack [25] that adapts to loss of information and partial failures of the beacon infrastructure.

\subsection{Distance Metric and Location Estimation}

The signature-based localization scheme used requires a set of base stations, i.e. beacon nodes, generally at fixed locations, to either transmit periodic beacon messages or receive signals from mobile nodes. Beacon nodes broadcast beacon messages at a range of transmission power and frequency levels. Using multiple transmission power levels and frequencies will cause a signal to propagate at various levels in its medium and therefore exhibit different characteristics at the receiver. The mobile nodes collect the signature, where signature is collection of tuples, and then use a distance metric to compare multiple locations and pick the one that best matches the observed signal strength.

Let $s$ be the received signature and let $R$ be the set of reference signatures. The location of a mobile node can be estimated as follows. The first step is to compute the signature distance, $D$, from $s$ to each reference signature $r$ which belongs to $R$. As for RADAR and MoteTrack the user can choose between two distance metrics for the performance and environmental behavior. The Euclidean distance is computed as:

$$
D=\sqrt{\left(x_{1}-y_{1}\right)^{2}+\left(x_{2}-y_{2}\right)^{2}+\left(x_{3}-y_{3}\right)^{2}}
$$

where we take the three dimensional way of the moving target using $X=\left(x_{1}, x_{2}, x_{3}\right)$ and $Y=\left(y_{1}, y_{2}, y_{3}\right)$. Where $x$ and $y$ are the distance metrics.

We use the Manhattan distance [25] metric as well:

$$
D(r, s)=\sum_{t \in T}\left|\operatorname{meanRSSI}(t)_{r}-\operatorname{meanRSSI}(t)_{s}\right|
$$

where $T$ is the set of signature tuples represented in both signatures, and meanRSSI $(t)_{r}$ is the mean RSSI value in the signature tuple $t$ appearing in signature $r$.

The mobile node first acquires its signature $s$ by listening to beacon messages, and then broadcasts $s$, requesting for the estimation of its location. Then one or more of the beacon nodes computes the signature distance between $s$ and their slice of the 
reference signature database. They report either a set of reference signatures to the mobile node, or directly compute the location of the mobile node. Usually the request is given to the beacon with the strongest RSSI, which is explained below.

The design we used here is strongest RSSI. In this design, we assume that the most relevant (closest in signal space) reference signatures are stored on the beacon node with the strongest signal. The beacon node sending the StrongestRSSI must contain the database of the particular area where the mobile node is requesting for the estimation of the location and it sends a request to the beacon node from which it received the strongest RSSI, and only that beacon node estimates the mobile node's location. As long as this beacon node stores an appropriate slice of the reference signature database, this should produce very accurate results. The main advantages are low communication overhead, accurate location estimates and communication cost is very low because only one reply is sent to the mobile node containing its location coordinates.

Therefore it is crucial that the reference signatures are distributed in an "optimal" fashion. In centralized fashion all the signatures are stored in the mobile mote and we wish to ensure that each reference signature is replicated across several beacon nodes in the event of beacon node failures. Here we use two algorithms for database distribution, which we refer to as stable marriage algorithm and the one referred from the MoteTrack which is greedy distribution algorithm.

The decentralized approach to RF-based localization is based on a network of battery-operated wireless nodes based on the 802.15.4 ZigBee standard [22].

\subsection{Decentralized Localization}

After we deal with the distance metric we need to concentrate on how the location of the moving target is estimated. This is done with the given set of signature distances $D$ from the above calculation algorithm of the metric distances. When the mobile node wants to track its location it sends a request to estimate the location. The beacon node, which contains the database, calculates the location of the mobile mote as a centroid, in which centroid is of set of signatures. Here we use the same approach as in MoteTrack where we consider the centroid of the set of signatures within some ratio of the nearest reference signature. Given a signature $s$, a set of reference signatures $R$, and the nearest signature $r$, we select all reference signatures $r$ belonging to $R$ that satisfy a mathematical function which is less than constant $c$. The given geographic centroid of the locations of this subset of reference signatures is then taken as the position of mobile node. Then this request from the mobile node is processed and the reply is sent to the mobile mote. Here the memory management, the fragmentation of the signature which is sent as the request for location estimation, the reassembly of this signature in the beacon mote and the queue system during the request for location and reply of the estimated location are the main ideas which are developed and expanded via our approach.

\subsection{Implementation and Data Collection Mode}

The developed decentralized localization and tracking system is programmed on the Arslogica-3Tec mote [15] platform using the TinyOS [10] operating system which are 
designed for low-power operation. It is relatively small and can be deployed unobtrusively in an indoor environment and, moreover, the developed system works on both CC1000 radio and CC2420 radio, which provides both programmable transmission power levels and direct sampling of received signal strength. We do not require any central server, which is one of the advantages of the system. A laptop connected to a mote is used to build the reference signature database, but thereafter the system is self-contained.

The developed system has a total code size for the beacon and mobile node software of about 2,500 lines of NesC [23] code and currently there is a front end GUI with normal display in JAVA which is still in the development phase. Since we need that each beacon node store a different set of reference signatures depending on the distribution mechanism used, we are concentrating primarily on the distribution of the reference signature.

Each reference signature consists of a tuple of the form \{SourceID, powerLevel, frequency, meanRSSI\}.

We have deployed the decentralized localization and tracking system over one floor of ArsLogica IT Laboratory. Our current installation consists of 20 beacon motes.

We collected a total of 250 reference signatures overall from throughout the laboratory. Each signature was collected for 1 minute, during which time every beacon node transmitted at a rate of $4 \mathrm{~Hz}$, each cycling through 4 frequency and 2 transmission power levels (from -20 to $10 \mathrm{dBm}$ in steps of $5 \mathrm{dBm}$ ).

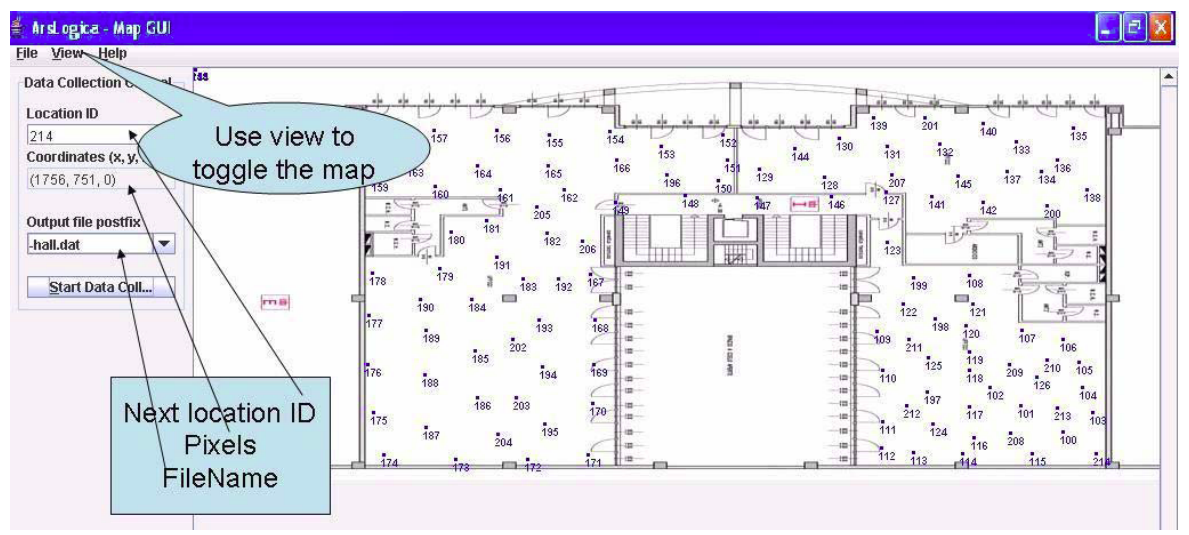

Fig. 3. The map of the IT laboratory with 250 Reference Signatures collected

A beacon message consists of a four-byte payload: 2 bytes for the source node ID, one byte representing the transmission power level and one byte representing the frequency level. We divided the collected signatures into two groups: the training data set (used to construct the reference signature database) and the testing data (used only for testing the accuracy of location tracking). Our analysis investigates the effects of a wide range of parameters including whether signatures are collected in a hallway or in a room, whether the room's door is open or closed, the time of the day (to account for solar radiation and building occupancy) and the use of different mobile nodes (to account for manufacturing differences). 


\subsection{System Accuracy Evaluation}

The system operation and accuracy can be improved by broadening the frequency band and increasing power, as it is shown in Figure $4(a, b)$ respectively. Figure $4 \mathrm{a}$ demonstrates the relationship between the error distance given in the $y$ axis and the frequency band in the $x$ axis. For predefined frequency 1, the accuracy decreases and the error rate increases up to $3.5 \mathrm{~m}$. When the frequency is gradually increased the accuracy improves and the error rate decreases. In other words, the system demonstrates the highest accuracy (less than $1 \mathrm{~m}$ ) at the frequency rate of 16.

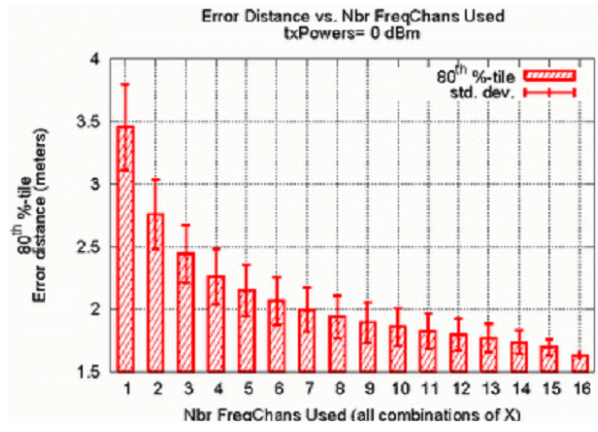

a)

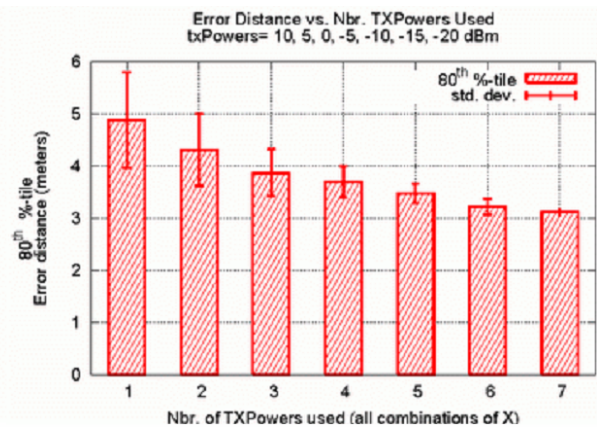

b)

Fig. 4. The graph showing the relationship between the system accuracy and the frequency $(\mathrm{MHz})$ variation $(\mathrm{a})$, and $\mathrm{TX}$ power $(\mu \mathrm{W})$ variation $(\mathrm{b})$

The main advantage of frequency increase is improved accuracy. However, there are some disadvantages, e.g., the longer period of signature collection and the larger size of signatures.

The graph presented in Figure $4 \mathrm{~b}$ depicts the accuracy achieved when the power varies from 1 to 7 . As this graph is derived from experiments, we can see that when the TX power decreases to its minimum, the error rate can reach its maximum. For example, when we had set power 2 and frequency 4, we got the average result from $2 \mathrm{~m}$ to $3 \mathrm{~m}$, which can be good enough for the system in which the RF technology is applied.

The main advantage of the TX power increase is improved accuracy. However, it has the same drawbacks (and larger power consumption) as we have mentioned above for the frequency increase.

\subsection{Selection of Reference Signature and Reference Algorithm}

The graphs presented in Figure $5(\mathrm{a}, \mathrm{b})$ show the error rate according to the type of algorithm applied for reference signature selection.

Our experiment was based mainly on the k-nearest algorithm. For the k-nearest algorithm (Figure 5a), the small values of $k$ are applicable for the location centroid computation, but the use of greater values results in significant errors. The relative thresholding graph (Figure 5b) is more accurate as it limits the set of locations considered in accordance with the signature distance metric. 


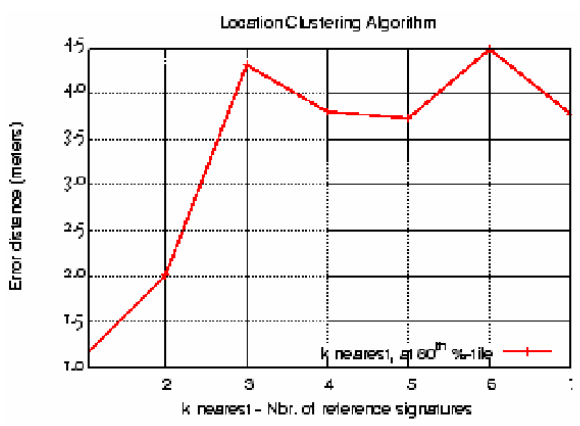

(a)

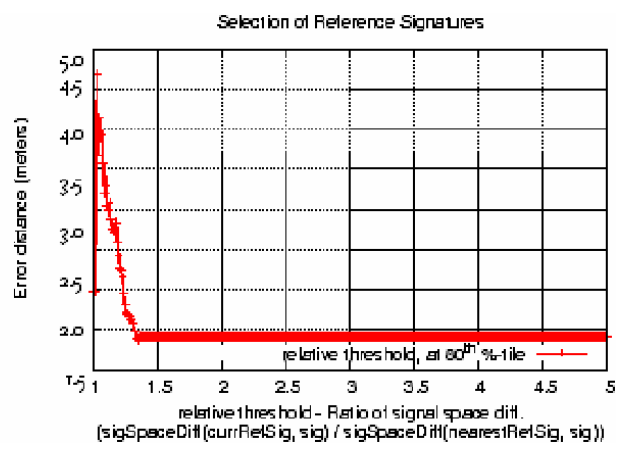

(b)

Fig. 5. The graphs of reference signature selection with k-nearest algorithm (a) and thresholdnearest algorithm (b)

\section{Conclusions and Future Work}

This paper describes the architecture of the self-powered module using environmental energy and the current status of its development. Besides, we have presented a decentralized localization and tracking system with the possibility of reference signature database distrbution. The experimental results have demonstrated its accuracy and tolerance to failure.

The robustness of the localization and tracking system can be improved by dividing its architecture into three modes, namely beacon, estimator and mobile motes. This solution is to reduce the beacon mote's overhead and improve the system performance.

In the very near future we intend to complete the self-powered module and test it together with the localization system for the potential use in scenario Paintball. However, the results achieved can be adapted for a variety of power-intensive applications where tracking and localization of mobile object is required.

\section{Acknowledgements}

This work was supported in part by a grant from ArsLogica, S.p.A. We would like to thank Fabrizio Stefani, Engineer (ArsLogica), and Prof. Alberto Montresor (University of Trento) for their valuable comments.

\section{References}

1. Ohta, Y., Sugano, M., Murata, M.: Autonomous localization method in wireless sensor networks. In: Pervasive Computing and Communication Workshops, March 8-12 (2005)

2. Torah, R.N., Tudor, M.J., Patel, K., Garcia, I.N., Beeby, S.P.: Autonomous low power microsystem powered by vibration energy harvesting. In: 6th annual IEEE Conference on Sensors, Atlanta, USA, October 28-31 (2007)

3. Raghunathan, V., Kansal, A., Hsu, J., Friedman, J., Srivastava, M.: Design considerations for solar energy harvesting wireless embedded systems. IEEE SPOTS (2005) 
4. Jiang, X., Polastre, J., Culler, D.: Perpetual environmentally powered sensor networks. IEEE SPOTS (April 2005)

5. Dutta, P., Hui, J., Jeong, J., Kim, S., Sharp, C., Taneja, J., Tolle, G., Whitehouse, K., Culler, D.: Trio: Enabling sustainable and scalable outdoor wireless sensor network deployments. IEEE SPOTS (2006)

6. Localization with GPS. From GPS Theory and Practice, 5th edn. (2005)

7. Polastre, Szewczyk, R., Culler, D.: Enabling ultra-low power wireless research. IEEE SPOTS (2005)

8. Dutta, P., Grimmer, M., Arora, A., Bibyk, S., Culler, D.: Design of a wireless sensor network platform for detecting rare, random and ephemeral events. IEEE IPSN (2005)

9. Mica2 wireless measurement system (November 2007), http://www.xbow.com/ Products/Product_pdf_files/Wireless_pdf/MICA2_Datasheet.pdf

10. TinyOS Programming, http://csl.stanford.edu/ pal/pubs/tinyosprogramming.pdf

11. Panasonic Industrial (June 2008), http: / / www . panasonic-industrial.com

12. Lorincz, K., Welsh, M.: A robust, decentralized approach to RF-based location tracking. Technical Report TR-19-04, Harvard University (2004)

13. Capkun, S., Hamdi, M., Hubaux: GPS-free positioning in mobile ad-hoc networks. System Sciences. In: Proceedings of the 34th Annual Hawaii International Conference, Fed. de Lausanne, Switzerland (2001)

14. Priyantha, N.B., Chakraborty, A., Balakrishnan, H.: The Cricket location-support system. In: Proceedings of the 6th annual International Conference on Mobile Computing and Networking (2000)

15. Using TREMATE. TRETEC S.r.l., Trento, Italy

16. van Greunen, J.: Services in sensor networks. Master thesis, University of California, Berkley (2003)

17. Harter, Hopper, A., Steggles, P., Ward, A., Webster, P.: The Anatomy of a Context-Aware Application. In: 5th ACM MOBICOM, Seattle, WA (August 1999)

18. Analog Devices (June 2008), http: / / www . analog . com

19. CC1000 Description (June 2008), http://focus.ti.com/lit/ds/symlink/cc1000.pdf

20. CC2420 Description (June 2008), http: / / enaweb. eng · yale. edu/ drupal/system/files/CC2420_Data_Sheet_1_4.pdf

21. Perpetuum (December 2007), http: / / www . perpetuum. co.uk

22. Ergen, S.C.: ZigBee/IEEE 802.15.4 Summary (2004)

23. David, G., Levis, P., Culler, D., Brewer, E.: nesC 1.1 Language Reference Manual (May 2003)

24. Nesscap, http://www. nesscap.com/

25. Lorincz, K., Welsh, M.: MoteTrack: A Robust, Decentralized Approach to RF-Based Location Tracking. In: Strang, T., Linnhoff-Popien, C. (eds.) LoCA 2005. LNCS, vol. 3479. Springer, Heidelberg (2005)

26. Bahl, P., Padmanabhan, V.N.: RADAR: An In-Building RF-based User Location and Tracking System. In: Infocom 2000 (2000)

27. Ekahau positioning engine (September 2008), http : / /www . ekahau . com

28. McNally, R., Arvind, D.: A Distributed Leaderless Algorithm for Location Discovery in Specknets. In: Kermarrec, A.-M., Bougé, L., Priol, T. (eds.) Euro-Par 2007. LNCS, vol. 4641. Springer, Heidelberg (2007)

29. MAXIM (June 2008), http : / /www . maxim-ic. com 